\title{
Hydrocephalus and craniosynostosis
}

\section{Giuseppe Cinalli, M.D., Christian Sainte-Rose, M.D., Eve Marie Kollar, M.D., Michel Zerah, M.D., Francis Brunelle, M.D., Paul Chumas, M.D., F.R.C.S. (SN), Eric Arnaud, M.D., Daniel Marchac, M.D., Alain Pierre-Kahn, M.D., and Dominique Renier, M.D.}

Cranio-Facial Surgery Unit, Department of Pediatric Neurosurgery, and Department of Pediatric Radiology, Hôpital Necker-Enfants Malades, Université René Descartes, Paris, France; and Department of Neurosurgery, Leeds General Infirmary, Leeds, England

Object. A retrospective study of 1727 cases of craniosynostosis was undertaken to determine the interrelationship between abnormal cerebrospinal fluid (CSF) hydrodynamics and craniosynostosis.

Methods. The patients were divided intwo two groups: nonsyndromic craniosynostosis and syndromic craniosynostosis. Cases of occipital plagiocephaly without suture synostosis and cases of shunt-induced craniosynostosis were excluded from the study. The majority of patients (1297) were treated surgically for their cranial deformity; $95 \%$ of these patients had a postoperative follow-up review lasting 5 years. Clinical and radiographic charts covering the time from presentation through the follow-up period were reviewed.

Conclusions. Abnormal intracranial CSF hydrodynamics was found in $8.1 \%$ of the patients $(3.4 \%$ of whom had received shunts and $4.5 \%$ of whom had not). Three types of CSF hydrodynamic disturbance were observed: progressive hydrocephalus with ventricular dilation, nonprogressive ventriculomegaly, and dilation of the subarachnoid spaces. Hydrocephalus occurred much more frequently in patients with syndromic craniosynostosis $(12.1 \%)$ than in those with isolated craniosynostosis $(0.3 \%)$. In fact, patients with kleeblattschädel exhibited hydrocephalus as a constant feature and patients with Crouzon's syndrome were far more likely to have hydrocephalus than those with other syndromes. In Apert's syndrome, ventricular dilation occurred very frequently, but it was almost always nonprogressive in nature. In most cases of syndromic craniosynostosis, venous sinus obstruction and/or chronic tonsillar herniation were found. Their role in the pathophysiology of hydrocephalus in craniosynostosis is discussed.

\section{Key Words * hydrocephalus * craniosynostosis * Crouzon's syndrome * Apert's syndrome * Chiari malformation * jugular foramen stenosis}

Both abnormal cerebrospinal fluid (CSF) hydrodynamics and craniosynostosis may result in increased intracranial pressure (ICP); however, they may have potentially opposing effects on cranial growth. The aim of this retrospective study was to investigate the interrelationship between these two processes. The association between hydrocephalus and craniosynostosis has been well documented and the incidence of hydrocephalus has been found to be between $4 \%$ and 10\%.[6-9,15,16,18,23,25,26,28] A significant 
increase in hydrodynamic problems among patients with syndromic craniosynostosis compared with patients with nonsyndromic craniosynostosis has been noted, $[4,9,23]$ and in the present study we examine the differing causative factors responsible for this.

There are two ways in which intracranial CSF volume may be increased: dilation of the ventricular cavities and dilation of the pericerebral spaces. Both of these may be observed either before or after craniofacial surgery, and both may be static or evolve to cause symptomatic intracranial hypertension. Each of these variations is correlated with types of craniosynostosis in the present article.

\section{CLINICAL MATERIAL AND METHODS}

The cases of 1727 children evaluated for craniosynostosis at the Hôpital Necker-Enfants Malades from 1976 to 1996 were retrospectively reviewed. The patients were divided into two groups: nonsyndromic craniosynostosis (695 scaphocephalies, 208 plagiocephalies, 241 trigonocephalies, 136 oxycephalies, 91 brachycephalies, 60 complex or unclassifiable craniosynostoses,[2] and 16 lambdoid suture synostoses) and syndromic craniosynostosis (86 Crouzon's, 77 Apert's, 40 Saethre-Chotzen, and 18 Pfeiffer's syndromes, 16 craniofrontonasal dysplasias, and 43 others). Three hundred forty-five cases of occipital plagiocephaly without suture synostosis that were believed to be caused by positional molding and patients with shunt-induced craniosynostosis were not included in this study.

In all cases the craniosynostosis was diagnosed after clinical and radiographic assessment (including plain x-ray films, cephalometric radiographs, and computerized tomography [CT] scans). The majority (70\%) of patients with syndromic craniosynostosis also underwent magnetic resonance (MR) imaging and some (40\%) of them underwent angiography or MR angiography as well. Magnetic resonance imaging and MR angiography have been performed in all cases of syndromic craniosynostosis since 1990. These examinations are performed at presentation in new cases or during outpatient clinic visits in patients seen for longer periods. Before 1990, angiography or sinusography was performed in all patients with hydrocephalus, papilledema, or increased intracranial hypertension at the time of ICP recording. The majority of patients (1297) were treated surgically for their cranial deformity; all of these patients underwent at least one CT study in the immediate postoperative period. Most surgical patients (95\%) were followed once a year for 5 years (mean follow up 4.2 years). Hydrocephalus was diagnosed by clinical progression (progressive head enlargement or intracranial hypertension) associated with one imaging study (CT or MR imaging) showing ventricular dilation and periventricular lucency in patients who exhibited hydrocephalus at presentation. In cases of patients who developed hydrocephalus during the follow-up period or after craniofacial surgery, hydrocephalus was diagnosed by two subsequent imaging studies showing increasing ventricular dilation and the appearance of periventricular lucency associated with clinical signs of increased intracranial hypertension (progressive head enlargement, intracranial hypertension, or tension at the craniectomy sites). A diagnosis of jugular foramen stenosis was made when a lateral imaging view showed a decreased diameter in both venous sinuses at the junction with the jugular vein that was associated with significant collateral venous drainage at the level of the dura of the posterior fossa or around the jugular bulb and/or significant transdiploic venous drainage (Fig. 1). 


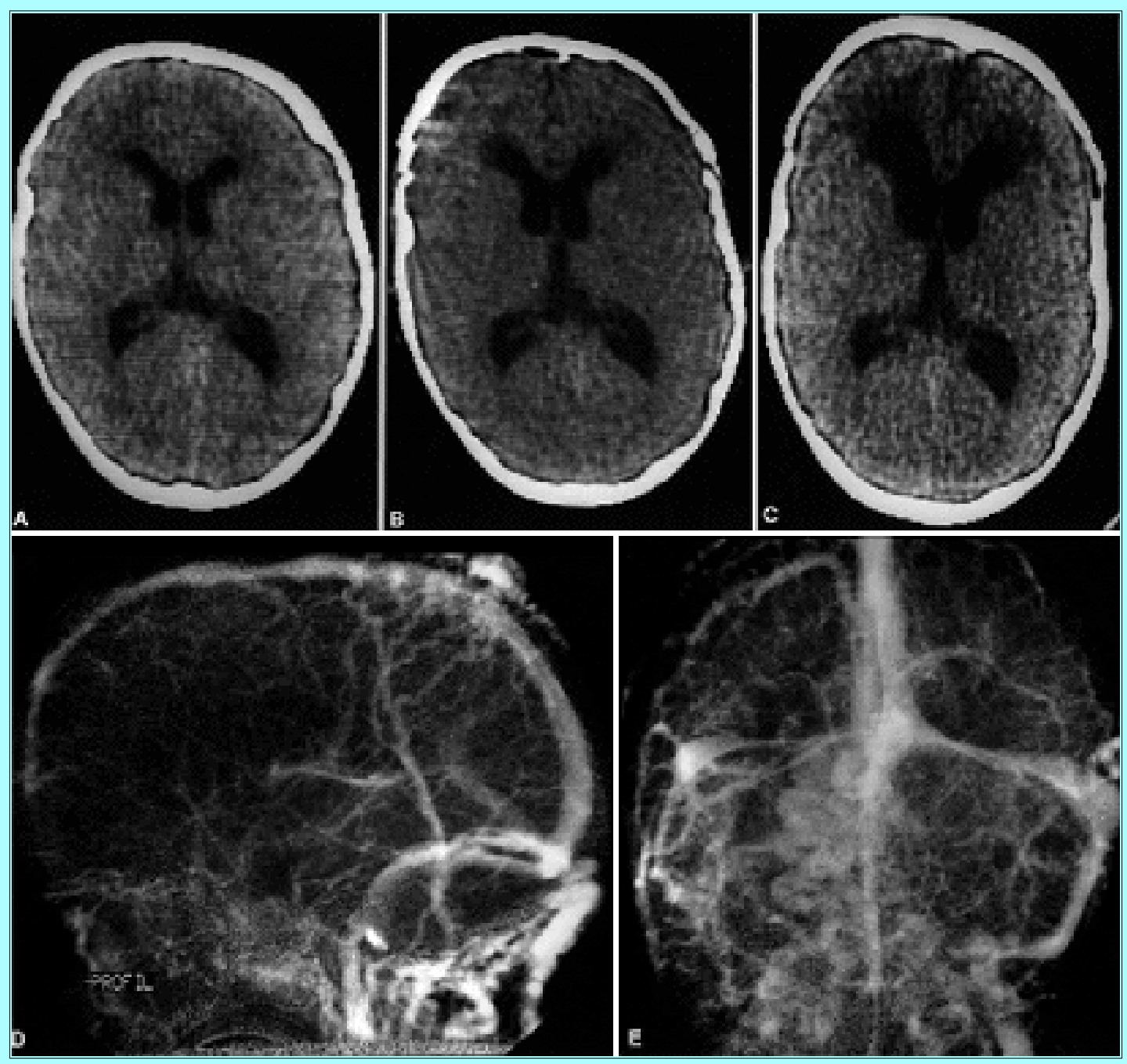

Fig. 1. Imaging data obtained in a patient with oxycephaly. A: Preoperative CT scan obtained when the patient was 5 years old. B: Postoperative CT scan obtained 2 weeks after forehead advancement. Note the increased ventricular size and the signs of transependymal resorption. C: A CT scan obtained 4 months after forehead advancement. There is a significant additional increase in ventricular size, which is more marked at the level of the frontal horns, with periventricular lucency. D: Angiogram, lateral view, showing stenosis of both jugular foramina. Note the significant transdiploic collateral circulation at the level of the sagittal sinus in the parietal region and at the level of the posterior fossa. E: Angiogram, posterior view, showing hypoplasia of both lateral sinuses, stenosis of both jugular foramina, and a marked collateral circulation.

\section{RESULTS}

A summary of the data is shown in Table 1. 


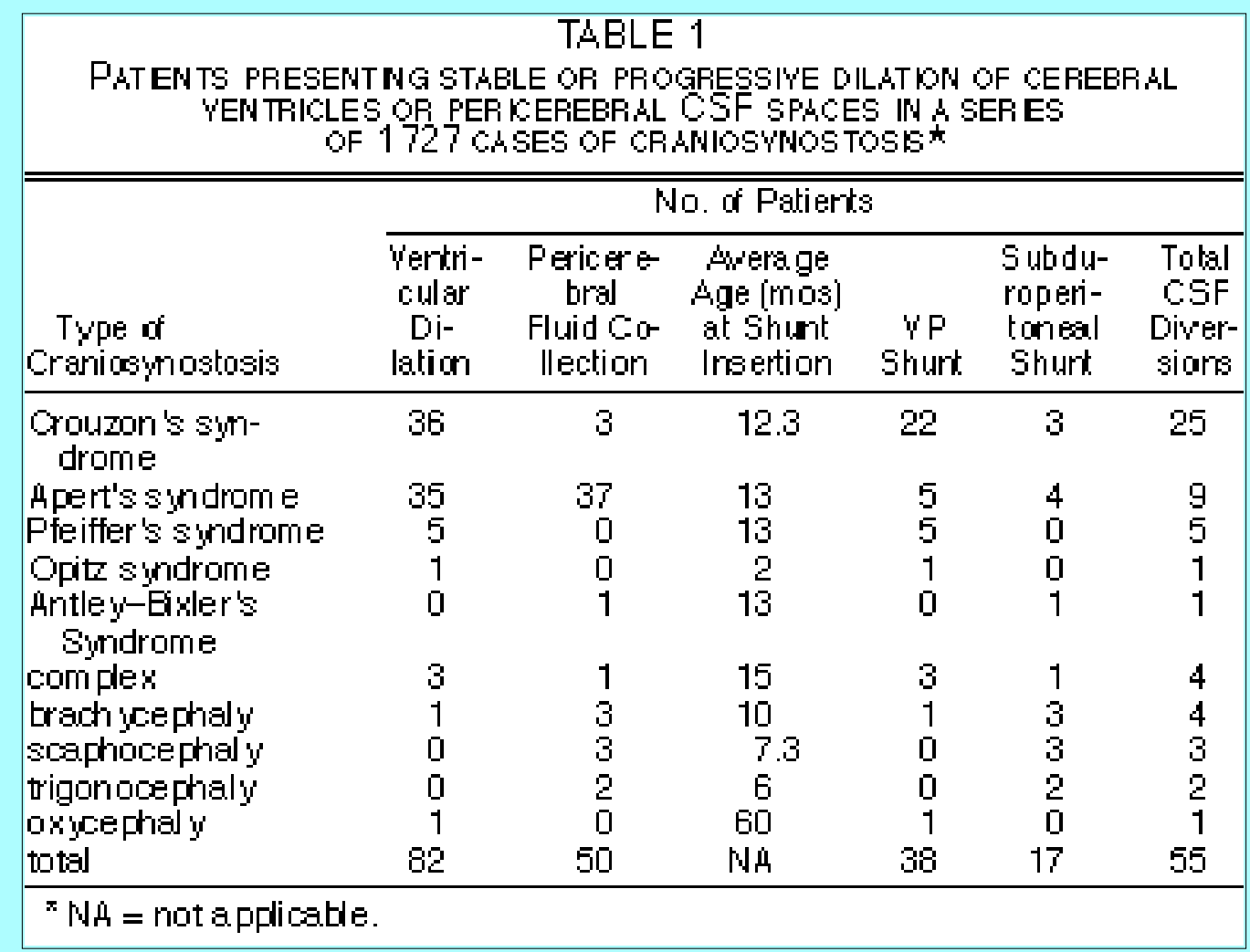

\section{Nonsyndromic Craniosynostosis}

Of the 1447 cases of nonsyndromic craniosynostosis, four patients $(0.28 \%)$ presented with progressive ventricular dilation that required shunt insertion (one patients with brachycephaly, one with oxycephaly, and two with complex craniosynostosis). The patient with brachycephaly required the placement of a ventriculoperitoneal (VP) shunt 15 months after undergoing craniofacial surgery. One patient with a complex case of craniosynostosis presented with chronic tonsillar herniation and severe stenosis of both jugular foramina. A lateral sinus-jugular vein bypass failed to control the hydrocephalus and she required placement of a VP shunt 1 year before she underwent craniofacial surgery. A second patient with complex craniosynostosis presented with chronic tonsillar herniation, jugular foramen stenosis, and severe ventricular dilation. This patient also required shunt insertion. The patient with oxycephaly had chronic tonsillar herniation and jugular foramen stenosis. A shunt was placed 5 months after this patient underwent craniofacial surgery (Fig. 1).

In the same group, nine patients $(0.6 \%)$ presented with a pericerebral fluid collection that required placement of a subduroperitoneal shunt (three patients with scaphocephaly, three with brachycephaly, two with trigonocephaly, and one with complex craniosynostosis). In all of these cases, the pericerebral fluid collection appeared or increased after the patient had undergone craniofacial surgery and required shunt placement because of symptoms of intracranial hypertension (three cases) or bone resorption at bulging craniectomy sites (six cases).

Four patients developed incidental hydrocephalus and, therefore, were excluded from the study: one patient with a myelomeningocele, one with an intraventricular hemorrhage, and two after contracting from meningitis. In all of these cases the craniosynostosis was already present at the time of the diagnosis of hydrocephalus; thus, it was possible to rule out a shunt-induced craniosynostosis.

\section{Syndromic Craniosynostosis}


In 280 cases of syndromic craniosynostosis, 34 patients (12.1\%) required surgical treatment for progressive ventricular dilation (22 cases of Crouzon's, five of Apert's, five of Pfeiffer's, and one case of Opitz syndromes, and one case of complex craniosynostosis) (Fig. 2). Eight patients (2.8\%) were treated for progressive pericerebral fluid collection by insertion of a subduroperitoneal shunt (four cases of Apert's, three of Crouzon's, and one case of Antley-Bixler syndromes). Forty-four patients showed evidence of nonprogressive ventriculomegaly (30 cases of Apert's and 14 of Crouzon's syndromes) and 33 cases (all cases of Apert's syndrome) presented with nonprogressive dilation of the subarachnoid or subdural spaces.

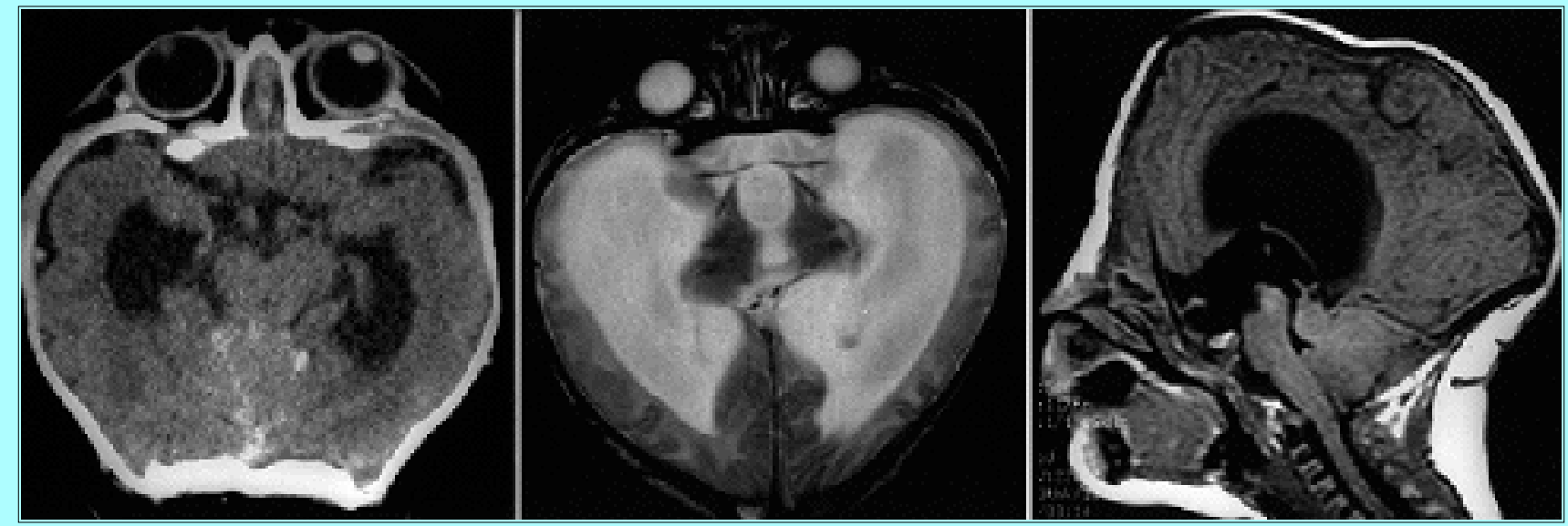

Fig. 2. Imaging data obtained in a patient with Opitz syndrome (C syndrome). Left: Computerized tomography scan obtained when the patient was 5 days old. Note the mild dilation of the temporal horns and severe cranial deformity. Center: Axial MR image obtained in the child at the age of 2 months, before shunt placement and cranial remodeling. Severe ventricular dilation is apparent. Right: Sagittal MR image obtained at the same time showing good visibility of the sylvian aqueduct and severe crowding of the posterior fossa without tonsillar herniation.

Crouzon's Syndrome. Hydrocephalus was present before procedures for frontal advancement or cranial vault remodeling were undertaken in 12 of 22 cases and required insertion of a shunt system in the patient at a mean age of 9 months (range 4-31 months). All these patients had coronal (four cases), sagittal (two cases), or both coronal and sagittal (three cases) sutures open at the time of the radiological diagnosis of ventricular enlargement. For three patients in whom the shunt placement had been performed at other institutions, original x-ray films were not available. In the other 10 patients, the hydrocephalus developed after the craniofacial surgery, with a mean delay of 6 months (range 9 days-12 months). All but one of these patients had a more severe form of craniosynostosis with early complete closure of coronal and sagittal sutures.

All of the patients suffering from Crouzon's syndrome with hydrocephalus were treated by placement of a VP shunt, except for two patients who were treated by a venous bypass (between the transverse sinus and the jugular vein) as previously described.[25] In one of these cases, this form of treatment was effective and stabilized the hydrocephalus (with a 13-year follow up), whereas in the other case it proved necessary to insert a VP shunt shortly after the operation because of persisting intracranial hypertension and despite the fact that the venous bypass was still functioning.[25] Magnetic resonance imaging revealed chronic tonsillar herniation in all but one patient. Thirteen of 16 angiographic studies revealed jugular foramen stenosis; the remaining three studies were normal. 
Fourteen patients with increased ventricular size did not require shunt insertion. In nine of them, mild-to-severe ventricular dilation was present preoperatively and remained stable after craniofacial surgery, whereas in five patients the ventricular size initially increased postoperatively and then stabilized.

Three patients underwent placement of a subduroperitoneal shunt for progressive enlargement of the pericerebral spaces with bulging craniectomy sites and bone resorption 1, 2, and 3 months, respectively, after frontal and vault remodeling (Fig. 3).

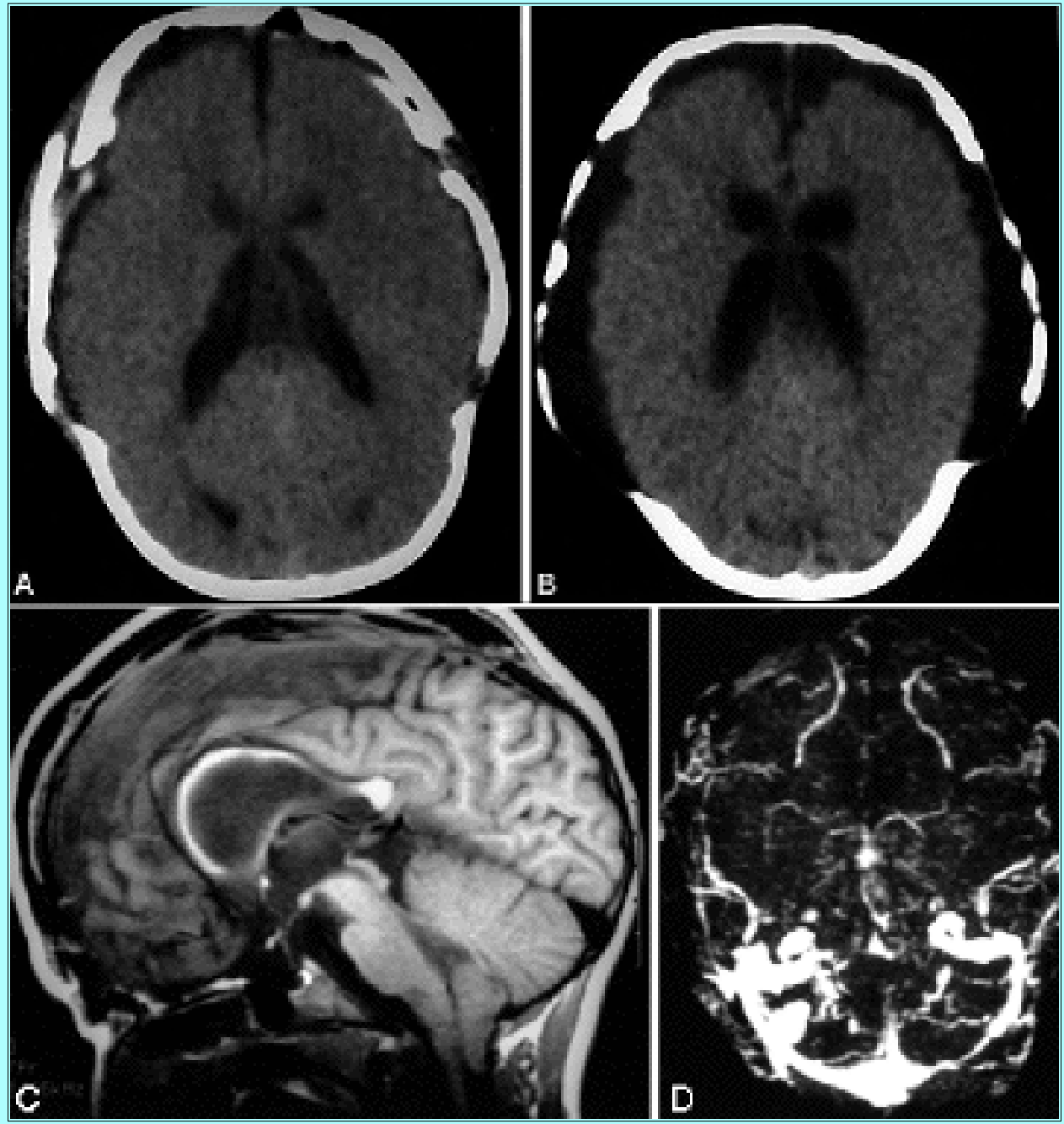

Fig. 3. Imaging data obtained in a patient with Crouzon's syndrome. A: Computerized tomography scan obtained when the patient was 20 months old, 1 week after forehead advancement and cranial vault remodeling. B: Computerized tomography scan obtained 2 months later. Note the mild increase in ventricular size and the marked dilation of the pericerebral spaces. The craniectomy sites are bulging, with severe bone resorption of the parietal flaps. C: Sagittal MR image showing chronic tonsillar herniation. D: Magnetic resonance angiography sequence displaying jugular foramen stenosis with hypoplasia of the left lateral sinus and significant collateral circulation at the level of the right sigmoid sinus.

Apert's Syndrome. In Apert's syndrome, progressive hydrocephalus that required shunt insertion was seen in only five cases. Nonprogressive ventricular dilation was present before craniofacial remodeling in 18 cases, whereas it appeared only after craniofacial surgery in 12 cases. Thirty-seven patients showed dilation of the pericerebral spaces after forehead advancement. This resolved spontaneously over several 
weeks in 33 cases, whereas subduroperitoneal shunt insertion was required in the remaining four cases (Fig. 4). Magnetic resonance imaging revealed chronic tonsillar herniation in only one patient not affected by hydrocephalus. Angiography was performed in 13 patients and revealed jugular foramen stenosis in seven cases. Of the five patients with hydrocephalus, arteriography was performed in only one case, revealing jugular foramen stenosis.
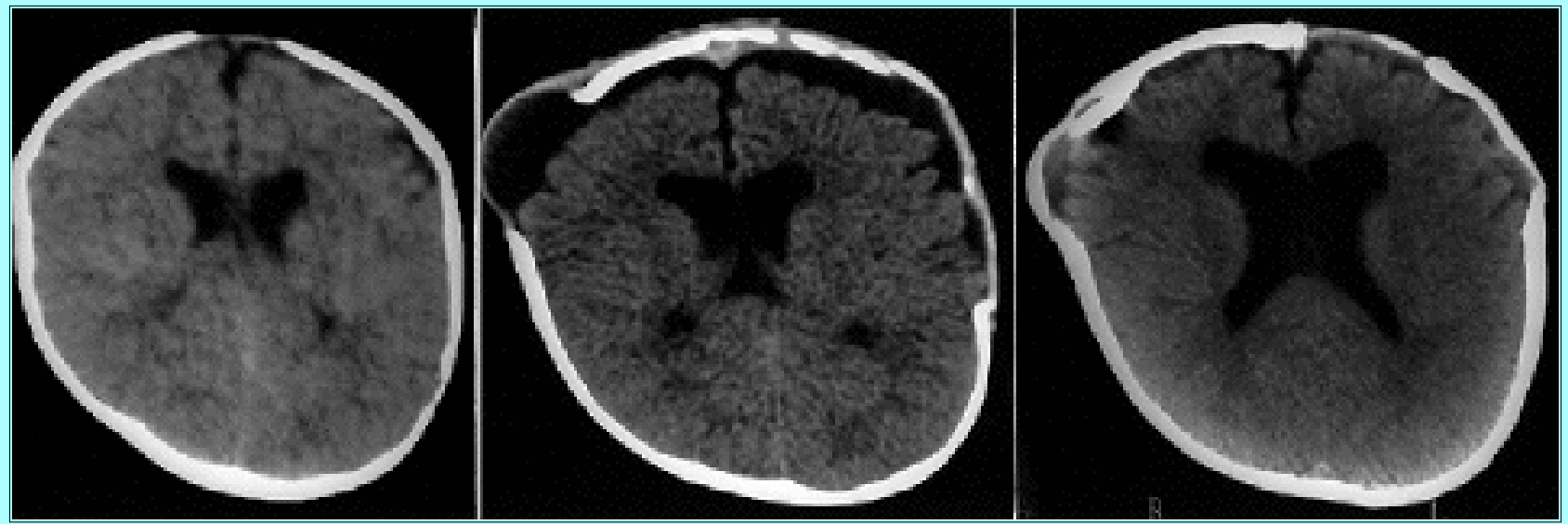

Fig. 4. Imaging data obtained in a patient with Apert's syndrome. Left: Preoperative CT scan obtained when the patient was 4 months old. Center: Computerized tomography scan obtained 2 months after forehead advancement. Note the persistence of a significant pericerebral fluid collection, bulging craniotomy sites with forehead deformity, and bilateral bone resorption. A subduroperitoneal shunt was placed in the patient a few days later. Right: Computerized tomography scan obtained 2 months after placement of the subduroperitoneal shunt. The pericerebral fluid collection has almost completely disappeared, but the craniotomy sites continue to bulge and the ventricular size has increased. A VP shunt was placed in the patient a few days later.

\section{DISCUSSION}

The series reported here includes all patients with craniosynostosis seen at our institution over a 20-year period. Analysis of the clinical data confirms that several types of disturbance in CSF hydrodynamics can be observed in patients affected by premature synostosis of the cranial sutures. In patients affected by nonsyndromic craniosynostosis, dilation of the cerebral ventricles occurs with the same frequency as that observed in the normal population.[1] All four instances es we observed occurred in patients with multisuture or complex craniosynostosis. Neuroradiological examination showed chronic tonsillar herniation and jugular foramen stenosis in three patients; the CT scans obtained at the level of the foramen magnum in the fourth patient were uninterpretable and no angiographic study was performed.

In contrast, ventriculomegaly (either stable or progressive) and/or dilation of the subarachnoid spaces was a relatively common finding in patients with syndromic craniofacial synostosis.

The role of venous hypertension as a pathophysiological mechanism in hydrocephalus in patients with craniosynostosis has previously been described.[25] Venous hypertension induced by jugular foramen stenosis results in a higher CSF pressure being required to maintain the CSF outflow balance.[8,25] The effect of this depends on the degree of cranial compliance. In infants and children with open sutures, increased ICP induces progressive head enlargement, resulting in dilation of ventricles and subarachnoid spaces. At the same time collateral venous pathways progressively develop and usually allow a new status quo to develop. A useful clinical model of this situation is seen in achondroplasia, in which jugular 
foramen stenosis (secondary to chondrocranial sutures synostosis) leads to rapidly progressive macrocrania with dilation of the ventricular and subarachnoid spaces in the first few months of life.[20] This stabilizes between the 4th month and the 2nd year of life[20] as a consequence of the formation of new collateral veins and because of cranial ossification. This explains the fact that patients with achondroplasia rarely require CSF diversion.[20]

However, if the venous obstruction occurs abruptly in this age group, the sudden rise in CSF pressure may result in progressive hydrocephalus and intracranial hypertension. Clinically, this situation is mirrored in the occasional case reports of hydrocephalus secondary to vena cava obstruction.[10,12,27] In contrast, in adults with nonexpansive skulls, venous hypertension results in increased ICP with normal-sized or small ventricles, as seen in some cases of pseudotumor cerebri.[21,25]

\section{Crouzon's Syndrome}

In Crouzon's syndrome, a complex succession of events takes place in the first 2 years of life. The progressive fusion of cranial base synchondroses produces alterations in the skull base[14] and stenosis of the jugular foramina. The resulting venous hypertension leads to increased CSF hydrostatic pressure. At the same time, the shape of the head will be determined by the pattern of premature fusion of the cranial vault sutures. This too may play an important role by potentially altering venous and CSF flow, as seen with early involvement of the lambdoid sutures in which crowding in the posterior fossa may increase venous outflow resistance by compressing the sigmoid sinus[13,17,19] and hindbrain herniation may increase CSF outflow resistance. $[4,11]$

Whatever the nature of the primary events that lead to increased CSF outflow resistance, in the present series clinical presentation could be divided into two groups: one group of patients with normal-sized or small ventricles that progressively increased after forehead advancement and cranial suture release, and a second group in which ventriculomegaly was noted prior to craniofacial surgery. Simultaneous or sequential involvement of the coronal and sagittal sutures may account for these differences in clinical presentation. In patients with early simultaneous involvement of these sutures a "pseudotumor-like" state may develop, with intracranial hypertension and normal-sized or small ventricles, due to a lack of skull vault compliance. After cranial remodeling and suture release, the ventricles would be free to enlarge and progressive hydrocephalus might occur (Fig. 1A-C). Patients presenting with a delayed or sequential synostosis of the coronal and sagittal sutures may present with varying degrees of ventricular dilation depending on the residual skull vault compliance (Fig. 5). Certainly, in the present series, all patients with Crouzon's syndrome who exhibited progressive hydrocephalus after craniofacial surgery were affected by a more severe form of the condition, with early fusion of the sagittal and both coronal sutures. Additionally, all patients noted to have ventricular enlargement prior to craniofacial surgery had open coronal or sagittal sutures at presentation (Fig. 5). 


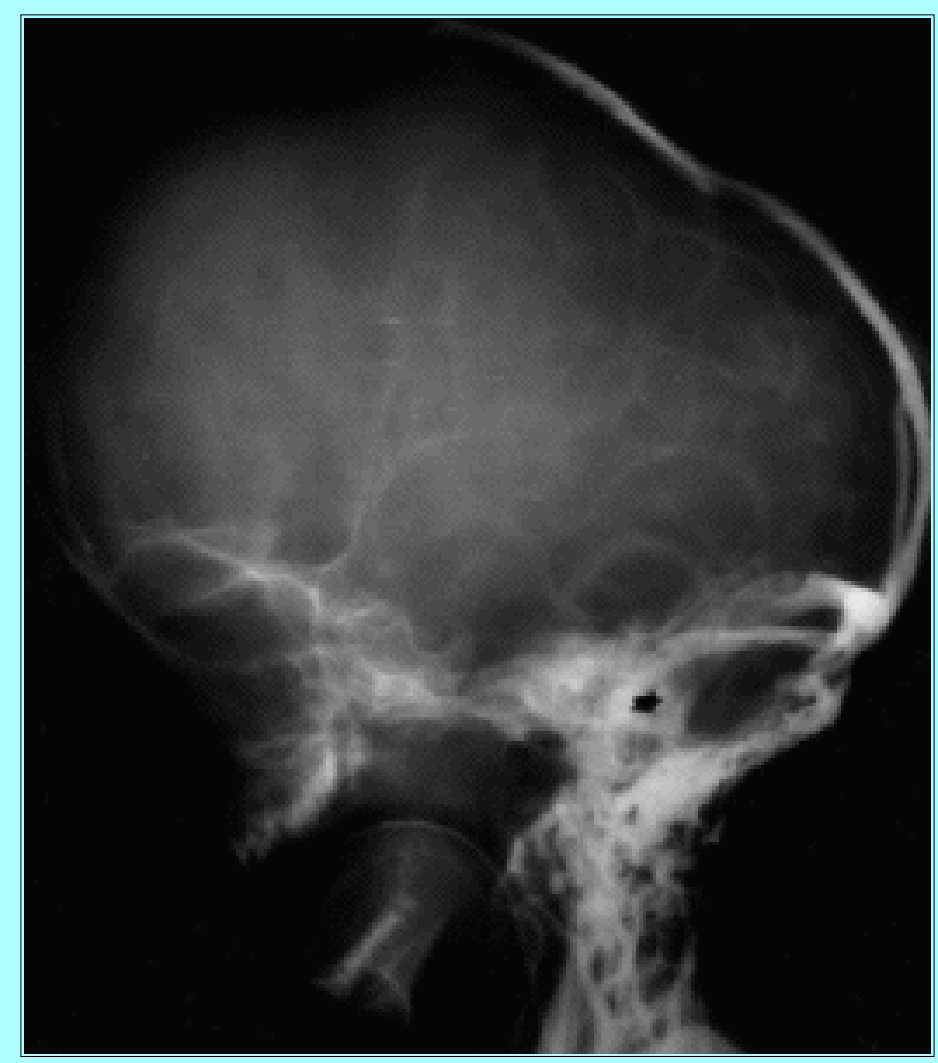

Fig. 5. Sinusography obtained in an 8-month-old patient with Crouzon's syndrome showing severe jugular foramen stenosis (arrow), chronic tonsillar herniation, and progressive ventricular dilation. Only the sagittal and lambdoid sutures are involved at this age. The coronal sutures are open and widely enlarged, allowing ventricular dilation to develop.

In some cases of mild preoperative ventriculomegaly there was no progression after craniofacial surgery. Similarly, there were other patients with normal ventricles preoperatively who showed mild, nonprogressive ventricular dilation after the craniofacial procedure. These patients were never symptomatic and required no intervention. It may be that these patients had less severe venous obstruction or developed better collateral vessels, allowing for a new status quo to be achieved.

The only exception in the present series was a patient with bicoronal and bilambdoid synostosis who had an open sagittal suture at the time of forehead advancement (age 2 months); angiography at this time revealed normal venous outflow. The patient underwent facial advancement at the age of 7 months and developed hydrocephalus at the age of 11 months. We postulate that this patient did not develop ventriculomegaly before the craniofacial surgery, in spite of the patent sagittal suture, because he was treated at such an early age ( 2 months), when the venous hypertension had not yet developed.

\section{Kleeblattschädel}

In the present series, cloverleaf skull deformity was observed in three cases of Pfeiffer's syndrome, one case of Crouzon's syndrome, and two cases of syndromic craniosynostosis that, to date, remain unclassified. This severe type of cranial abnormality has all the factors potentially responsible for ventricular dilation and, therefore, it is not surprising that this group of patients is at particular risk of developing hydrocephalus.[3,26,28,29] Perinatal fusion of multiple sutures results in a significant reduction in intracranial volume, especially of the posterior fossa.[29] Venous outflow is severely impaired, with significant stenosis or obstruction of the jugular foramina and development of a collateral circulation based at the level of the occipital foramen or through the calvaria via the subcutaneous 
tissues.[28] Significant hindbrain herniation has been found in all of the patients (in the present series and in others[3,10,28,29]) along with severe crowding in the posterior fossa, resulting in brainstem compression and deformation of the fourth ventricle. Occasionally, we have observed hydrocephalus and hindbrain herniation develop some months after birth.[3]

The question arises as to whether the hydrocephalus is the consequence of the progressive obliteration of the CSF spaces due to posterior fossa changes[29] or whether it is induced by venous hypertension related to jugular foramen stenosis.[25] As with Crouzon's syndrome, the pathophysiological mechanisms are probably multifactorial and there is evidence in the literature of both communicating and obstructive hydrocephalus being associated with the morphological appearance of cloverleaf skull.[26]

\section{Apert's Syndrome}

Because Apert's syndrome differs from Crouzon's syndrome in several aspects, it is not surprising that the pathophysiological mechanism of the dilation of the CSF spaces also differs. Although the bicoronal synostosis may be severe and may be seen very early,[4,14] sagittal and lambdoid suture involvement is rare and usually late. In fact, it is not uncommon for the sagittal suture to remain abnormally wide for several years after birth in children with Apert's syndrome.[5] Additionally, fusion of the cranial base synchondroses occurs later than in Crouzon's syndrome with less cranial base deformity[14] and, hence, less severe venous hypertension. Magnetic resonance imaging studies have also noted cerebral structural changes with frequent abnormalities of the septum pellucidum and the corpus callosum.[22] These differences result in a normal-sized or large posterior fossa[4,24] without hindbrain herniation.[4,22] Nonetheless, jugular foramen stenosis does occur, although in our angiographic studies, this was less frequent and less severe than in Crouzon's syndrome. This moderate venous hypertension, in the setting of large open sutures, may explain the fact that ventricular dilation is a frequent event in Apert's syndrome, but is rarely progressive.

\section{CONCLUSIONS}

Hydrocephalus is a rare event in cases of nonsyndromic craniosynostosis and never occurs in cases of single-suture synostosis, other than by coincidence. In contrast, hydrocephalus is a relatively frequent finding in cases of syndromic craniostenosis, especially in those of Crouzon's and Apert's syndromes, and is a constant finding in cases of kleeblattschädel deformity. Jugular foramen stenosis and crowding of the posterior fossa are probably the two main factors responsible for hydrocephalus in cases of Crouzon's syndrome and kleeblattschädel deformity. Ventricular dilation may reflect a more widespread brain malformation in Apert's syndrome, although jugular foramen stenosis can occasionally induce progressive hydrocephalus.

Significant pericerebral fluid collections that require placement of a shunt are occasionally observed postoperatively in cases of nonsyndromic craniosynostosis. However, dilation of the subarachnoid spaces, both pre- and postoperatively, is a frequent finding in Apert's syndrome and might be a result of a primary brain abnormality that causes slow brain reexpansion after cranial vault remodeling.

\section{Acknowledgment}

The authors gratefully acknowledge Bertrand Best for his invaluable help.

\section{References}


1. Bondurant CP, Jimenez DF: Epidemiology of cerebrospinal fluid shunting. Pediatr Neurosurg 23:254-259, 1995

2. Chumas PD, Cinalli G, Arnaud E, et al: Classification of previously unclassified cases of craniosynostosis. J Neurosurg 86:177-181, 1997

3. Cinalli G, Chumas P, Arnaud E, et al: Occipital remodeling and suboccipital decompression in severe craniosynostosis associated with tonsillar herniation. Neurosurgery (In press, 1998)

4. Cinalli G, Renier D, Sebag G, et al: Chronic tonsillar herniation in Crouzon's and Apert's syndrome: the role of premature synostosis of the lambdoid suture. J Neurosurg 83:575-582, 1995

5. Cohen MM Jr, Kreiborg S: Suture formation, premature suture fusion, and sutural default zones in Apert syndrome. Am J Med Genet 62:339-344, 1996

6. Collmann H, Sörensen N, Krauss J, et al: Hydrocephalus in craniosynostosis. Childs Nerv Syst 4:279-285, 1988

7. Fishman MA, Hogan GR, Dodge PR: The concurrence of hydrocephalus and craniosynostosis. $\mathbf{J}$ Neurosurg 34:621-629, 1971

8. Francis PM, Beals S, Rekate HL, et al: Chronic tonsillar herniation and Crouzon's syndrome. Pediatr Neurosurg 18:202-206, 1992

9. Golabi M, Edwards MSB, Ousterhout DK: Craniosynostosis and hydrocephalus. Neurosurgery 21:63-67, 1987

10. Haar FL, Miller CA: Hydrocephalus resulting from superior vena cava thrombosis in an infant. Case report. J Neurosurg 42:597-601, 1975

11. Hayward R, Harkness W, Kendall B, et al: Magnetic resonance imaging in the assessment of craniosynostosis. Scand J Plast Reconstr Hand Surg 26:293-299, 1992

12. Hooper R: Hydrocephalus and obstruction of the superior vena cava in infancy. Clinical study of the relationship between cerebrospinal fluid pressure and venous pressure. Pediatrics 28:792-799, 1961

13. Kinal ME: Hydrocephalus and the dural venous sinuses. J Neurosurg 19:195-201, 1962

14. Kreiborg S, Marsh JL, Cohen MM Jr, et al: Comparative three-dimensional analysis of CT-scans of the calvaria and cranial base in Apert and Crouzon syndromes. J Craniomaxillofac Surg 21:181-188, 1993

15. Lundar T, Nornes H: Steady-state lumbar infusion tests in the management of children with craniosynostosis. Childs Nerv Syst 7:31-33, 1991

16. Marchac D, Renier D: "Le front flottant." Traitement précoce des facio-craniosténoses. Ann Chir Plast 24:121-126, 1979

17. Martins AN, Kobrine AI, Larsen DF: Pressure in the sagittal sinus during intracranial hypertension in man. J Neurosurg 40:603-608, 1974

18. Noetzel MJ, Marsh JL, Palkes H, et al: Hydrocephalus and mental retardation in craniosynostosis. $\mathbf{J}$ 
Pediatr 107:885-892, 1985

19. Osterholm JL: Reaction of the cerebral venous sinus system to acute intracranial hypertension. J Neurosurg 32:654-659, 1970

20. Pierre-Kahn A, Hirsch JF, Renier D, et al: Hydrocephalus and achondroplasia. A study of 25 observations. Childs Brain 7:205-219, 1980

21. Ray BS, Dunbar HS: Thrombosis of the dural venous sinuses as a cause of "pseudotumor cerebri." Ann Surg 134:376-386, 1951

22. Renier D, Arnaud E, Cinalli G, et al: Prognosis for mental function in Apert's syndrome. J Neurosurg 85:66-72, 1996

23. Renier D, Sainte-Rose C, Marchac D, Hirsch JF: Intracranial pressure in craniostenosis. J Neurosurg 57:370-377, 1982

24. Richtsmeier JT: Comparative study of normal, Crouzon, and Apert craniofacial morphology using finite element scaling analysis. Am J Phys Anthropol 74:473-493, 1987

25. Sainte-Rose C, LaCombe J, Pierre-Kahn A, et al: Intracranial venous sinus hypertension: cause or consequence of hydrocephalus in infants? J Neurosurg 60:727-736, 1984

26. Shiroyama Y, Ito H, Yamashita T, et al: The relationship of cloverleaf skull syndrome to hydrocephalus. Childs Nerv Syst 7:382-385, 1991

27. Stewart DR, Johnson DG, Myers GG: Hydrocephalus as a complication of jugular catheterization during total parenteral nutrition. J Pediatr Surg 10:771-777, 1975

28. Thompson DNP, Hayward RD, Harkness WJ, et al: Lessons from a case of kleeblattschädel. Case report. J Neurosurg 82:1071-1074, 1995

29. Venes Jl: Arnold-Chiari malformation in an infant with Kleeblattschädel: an acquired malformation? Neurosurgery 23:360-362, 1988

Manuscript received June 11, 1997.

Accepted in final form September 26, 1997.

Address reprint requests to: Giuseppe Cinalli, M.D., Service de Neurochirurgie, Hôpital Necker-Enfants Malades, 149 rue de Sevres, 75015 Paris, France. 